\title{
ON QUASI-NORMABLE ECHELON SPACES
}

\author{
by M. VALDIVIA
}

(Received 27th June 1979, revised 16th January 1980)

In this paper we prove that if the strong dual of an echelon space fulfils the Mackey convergence condition the echelon space is quasi-normable. Also we give a characterisation of the quasi-normable echelon spaces and we deduce that every non-quasinormable echelon space is the strong dual of a non-complete (LB)-space.

The vector spaces used here are defined on the field $K$ of real or complex numbers. By the word "space" we mean a "locally convex space". Given a dual pair $\langle E, F\rangle$, $\sigma(E, F), \mu(E, F)$ and $\beta(E, F)$ are the weak, Mackey and strong topologies on $E$, respectively. Given a space $H, H^{\prime}$ is its topological dual. If $B$ is a bounded absolutely convex subset of $H, H_{B}$ is the normed space on the linear hull of $B$ normed by the Minkowski functional of $B$. A sequence $\left(x_{n}\right)$ in $H$ converges to $x$ in the sense of Mackey if there is an absolutely convex and bounded subset of $H, A$, such that $x$ and $x_{n}$ belong to $H_{A}, n=1,2, \ldots$, and $\left(x_{n}\right)$ converges to $x$ in $H_{A} . H$ verifies the Mackey convergence condition if every convergent sequence in $H$ converges in the sense of Mackey. $H$ verifies the strict Mackey convergence condition if for every bounded set $M$ in $H$ there exists a bounded absolutely convex set $B$ in $H$ such that $M$ is bounded in $H_{B}$ and the topology of $H_{B}$ coincides with topology of $H$ on $M$. If $H$ is quasi-barrelled, $H$ is quasi-normable, $(\mathbf{1}$, p. 106$)$, if $H^{\prime}\left[\beta\left(H^{\prime}, H\right)\right]$ verifies the strict Mackey convergence condition.

In the sequel $\lambda$ will denote an echelon space and $\lambda^{x}$ its $\alpha$-dual. Let us suppose that the echelons which define $\lambda$

$$
\alpha^{(n)}=\left(a_{1}^{(n)}, a_{2}^{(n)}, \ldots, a_{p}^{(n)}, \ldots\right), \quad n=1,2, \ldots,
$$

are positive, form an increasing sequence of vectors and for each index $p$ there is an index $q$ such that $a_{p}^{(q)} \neq 0$, (see (3)). Let

$$
B_{n}=\left\{\alpha=\left(a_{1}, a_{2}, \ldots, a_{p}, \ldots\right):\left|a_{p}\right| \leqq a_{p}^{(n)}, p=1,2, \ldots\right\}
$$

We put $\lambda_{\alpha}^{x}$ for the linear hull of $B_{n}$ endowed with the closed unit ball $B_{n}$. The norm of $\lambda_{\alpha^{(n)}}^{x}$ is $\|\cdot\|$.

$\varphi$ is the space of all sequences with only finitely many non-zero coordinates. By $\varphi^{(1)}$ we represent the subspace of $\lambda^{x}\left[\beta\left(\lambda^{x}, \lambda\right)\right]$ of all the limits in the sense of Mackey of sequences in $\varphi$.

If $P$ is a subset of the set $\mathbb{N}$ of positive integers, let $\lambda^{x}(P)$ be the subspace of $\lambda^{x}\left[\beta\left(\lambda^{x}, \lambda\right)\right]$ of the vectors with vanishing coordinates corresponding to indices outside $P$. 
Lemma. The space $\varphi^{(1)}$ is complete if and only if there is for each $p \in \mathbb{N}$ an integer $q>p$ such that, if $P$ is an arbitrary subset of $\mathbb{N}$ which verifies

$$
a_{n}^{(p)} \neq 0, \quad n \in P, \quad \text { inf }\left\{\frac{a_{n}^{(p)}}{a_{n}^{(q)}}: n \in P\right\} \neq 0
$$

then

$$
\inf \left\{\frac{a_{n}^{(p)}}{a_{n}^{(r)}}: n \in P\right\} \neq 0, \quad r=q+1, q+2, \ldots
$$

Proof. Suppose that there is a $p \in \mathbb{N}$ such that, for each $q>p$, we can find an integer $r, r>q$, and a subset $P$ of $\mathbb{N}$, such that

$$
a_{n}^{(p)} \neq 0, \quad n \in P, \quad \text { inf }\left\{\frac{a_{n}^{(p)}}{a_{n}^{(q)}}: n \in P\right\} \neq 0, \quad \text { inf }\left\{\frac{a_{n}^{(p)}}{a_{n}^{(r)}}: n \in P\right\}=0 .
$$

We can select in $P$ a sequence $n_{1}<n_{2}<\ldots<n_{s}<\ldots$, so that

$$
\begin{aligned}
& \lim \frac{a_{n_{s}}^{(p)}}{a_{n_{1}}^{(q)}}=a_{q} \neq 0, \\
& \lim \frac{a_{n_{1}}^{(p)}}{a_{n_{1}}^{(r)}}=0 .
\end{aligned}
$$

The vector

$$
\gamma=\left(c_{1}, c_{2}, \ldots, c_{n}, \ldots\right)
$$

of $\lambda^{x}$ defined by $c_{n}=0$, if $n \neq n_{s}, c_{n}=a_{n}^{(p)}$, if $n=n_{s}, s=1,2, \ldots$, lies in $\lambda_{\alpha}^{x}(p)$.

Let $\varphi_{n}$ be the closure of $\varphi \cap \lambda_{\alpha^{(n)}}^{x}$ in $\lambda_{\alpha^{(n)}}^{x}$ with the topology induced by $\lambda_{\alpha^{(n)} \text {. }}^{x}$ According to (1), $\gamma$ does not belong to $\varphi_{a}$, and by (2), $\gamma$ lies in $\varphi_{r}$. Therefore

$$
\varphi_{r} \cap \lambda_{\alpha}^{x} \neq \varphi_{q} \cap \lambda_{\alpha^{(D)}}^{x}
$$

and, consequently,

$$
\left(\varphi_{n} \cap \lambda_{\alpha}^{x}{ }^{(0)}\right)_{n=p}^{\infty}
$$

is an increasing non-stationary sequence of closed subspaces of $\lambda_{\alpha}^{x}(p)$. Applying the theorem of Baire to the Banach space $\lambda_{\alpha^{(p)}}^{x}$ it results that

$$
\cup\left\{\varphi_{n} \cap \lambda_{\alpha^{(p)}}^{x}: n=1,2, \ldots\right\}=\varphi^{(1)} \cap \lambda_{\alpha^{(p)}}^{x}
$$

is a non-closed subspace of $\lambda_{\alpha}^{x}$ and therefore $\varphi^{(1)}$ is not complete.

Suppose now that the conditions of the lemma are verified. If $\varphi^{(1)}=\lambda^{x}$ then $\varphi^{(1)}$ is complete. If $\varphi^{(1)}$ is different from $\lambda^{x}$ we take a vector $\beta=\left(b_{1}, b_{2}, \ldots, b_{n}, \ldots\right)$ in $\lambda^{x}$ which is not in $\varphi^{(1)}$. Let $p \in \mathbb{N}$ be such that $\beta \in \lambda_{\alpha}^{x}(\mathrm{p})$. We obtain an integer $q>p$ so that, if $P$ is an arbitrary subset of $\mathbb{N}$ which satisfies

$$
a_{n}^{(p)} \neq 0, \quad n \in P, \quad \text { inf }\left\{\frac{a_{n}^{(p)}}{a_{n}^{(q)}}: n \in P\right\} \neq 0
$$


then

$$
\inf \left\{\frac{a_{n}^{(p)}}{a_{n}^{(r)}}: n \in P\right\} \neq 0, \quad r=q+1, q+2, \ldots
$$

Since $\beta \in \lambda_{\alpha^{(p)}}^{x}$ and $\beta \notin \varphi_{q}$ we can find a sequence of positive integers

$$
n_{1}<n_{2}<\ldots<n_{s}<\ldots
$$

so that

$$
a_{n_{2}}^{(p)} \neq 0, \quad s=1,2, \ldots
$$

and

$$
\inf \left\{\frac{\left|b_{n_{3}}\right|}{a_{n_{2}}^{q}}: s=1,2, \ldots\right\} \neq 0 .
$$

There exists a positive real number $b$ such that

$$
\left|b_{n_{3}}\right| \leqq b a_{n_{*}}^{(p)}, \quad s=1,2, \ldots
$$

Then

$$
\inf \left\{\frac{a_{n_{s}}^{(p)}}{a_{n_{1}}^{(q)}}: s=1,2, \ldots\right\} \geqq \inf \left\{\frac{\left|b_{n_{1}}\right|}{b a_{n_{2}}^{(q)}}: s=1,2, \ldots\right\} \neq 0
$$

and therefore

$$
\inf \left\{\frac{a_{n_{2}}^{(p)}}{a_{n_{2}}^{(r)}}: s=1,2, \ldots\right\} \neq 0, \quad r=q+1, q+2, \ldots
$$

Using a diagonal process we can obtain a subsequence $\left(m_{s}\right)$ of $\left(n_{s}\right)$ so that

$$
\begin{aligned}
& \lim _{s \rightarrow \infty} \frac{a_{m_{s}}^{(p)}}{a_{m_{s}}^{(r)}}=a_{r} \neq 0, \quad r=q, q+1, \ldots \\
& \lim _{s \rightarrow \infty} \frac{b_{m_{s}}}{a_{m_{s}}^{(q)}}=c \neq 0 .
\end{aligned}
$$

Let $\lambda_{1}^{x}$ be the subspace of $\lambda^{x}\left[\beta\left(\lambda^{x}, \lambda\right)\right]$ consisting of all the sequences with the $n$th coordinate vanishing, for $n \in \mathbb{N}, n \neq m_{s}, s=1,2, \ldots$ Let $\lambda_{2}^{x}$ be the subspace of $\lambda^{x}\left[\beta\left(\lambda^{x}, \lambda\right)\right]$ consisting of all the sequences with the $m_{s}$ th coordinate vanishing, $s=1,2, \ldots$ Then $\lambda^{x}\left[\beta\left(\lambda^{x}, \lambda\right)\right]=\lambda_{1}^{x} \oplus \lambda_{2}^{x}$.

From (3) and (4) we obtain that $\lambda_{1}^{x}$ is topologically isomorphic to $l^{\infty}$ and the projection of $\beta$ onto $\lambda_{1}^{x}$ along $\lambda_{2}^{x}$ is not in the closure of $\varphi \cap \lambda_{1}^{x}$ in $\lambda_{1}^{x}$. On the other hand, since $\varphi^{(1)}$ is a normal space it follows in a straightforward way that the projection of the completion of $\varphi^{(1)}$ onto $\lambda_{1}^{x}$ along $\lambda_{2}^{x}$ coincides with the closure of $\varphi \cap \lambda_{1}^{x}$ in $\lambda_{1}^{x}$ and therefore $\beta$ does not belong to the completion of $\varphi^{(1)}$ and so $\varphi^{(1)}$ is complete.

Theorem 1. If $\varphi^{(1)}$ is complete, then $\lambda\left[\mu\left(\lambda, \lambda^{x}\right)\right]$ is quasi-normable.

Proof. Given a bounded absolutely convex subset $A$ of $\lambda^{x}\left[\beta\left(\lambda^{x}, \lambda\right)\right]$, we can find a positive integer $p$ such that $A$ is a bounded set in $\lambda_{\alpha^{(p)}}^{x}$. Let $q$ be a positive integer, $q>p$, which verifies the conditions of the lemma. 
Let us suppose that $\beta\left(\lambda^{x}, \lambda\right)$ and the topology of $\lambda_{\alpha}^{x}(a)$ do not coincide on $A$. Therefore there is a positive number $c$ and a net in $A$

$$
\left\{\left(x_{i}^{(j)}\right): j \in J, \geqq\right\}
$$

which converges to the origin in $\lambda^{x}\left[\beta\left(\lambda^{x}, \lambda\right)\right]$ such that

$$
\left\|\left(x_{i}^{(j)}\right)\right\|_{q}>c, \quad j \in J \text {. }
$$

If $j \in J$, we have that

$$
\left\|\left(x_{i}^{(j)}\right)\right\|_{q}=\sup \left\{\frac{\left|x_{i}^{(j)}\right|}{a_{i}^{(q)}}: a_{i}^{(q)} \neq 0\right\} .
$$

Consequently, there exists a positive integer $m_{j}$ so that

$$
a_{m_{1}}^{(q)} \neq 0, \frac{\left|x_{m_{1}}^{(j)}\right|}{a_{m_{1}}^{(q)}}>c .
$$

For a suitable positive real number $b$

$$
\left|x_{m_{i}}^{(j)}\right| \leqq b a_{m_{i}}^{(p)}, \quad j \in J,
$$

hence

$$
b \geqq \frac{b a_{m_{1}}^{(p)}}{a_{m_{1}}^{(q)}} \geqq \frac{\left|x_{m_{1}}^{(j)}\right|}{a_{m_{1}}^{(q)}}>c,
$$

and therefore

$$
\inf \left\{\frac{a_{m_{1}}^{(p)}}{a_{m_{i}}^{(q)}}: j \in J\right\} \neq 0
$$

Then, by the lemma,

$$
\inf \left\{\frac{a_{m_{1}}^{(p)}}{a_{m_{1}}^{(r)}}: j \in J\right\} \neq 0, \quad r=q, q+1, \ldots
$$

If $M$ is the set $\left\{m_{j}: j \in J\right\}$, the space $\lambda^{x}\left[\beta\left(\lambda^{x}, \lambda\right)\right]$ is the topological direct sum of $\lambda^{x}(M)$ and $\lambda^{x}(N \sim M)$. Condition (7) shows that $\lambda^{x}(M)$ is topologically isomorphic to a Banach space. The projection of the net (5) on $\lambda^{x}(M)$ along $\lambda^{x}(N \sim M)$ converges to the origin in $\lambda^{x}(M)$ and, therefore, given a positive number $\varepsilon<c$, there exists a $j_{0} \in J$ such that, if $j \geqq j_{0}$,

$$
\sup \left\{\frac{\left|x_{i}^{(j)}\right|}{a_{i}^{(q)}}: i \in M\right\}<\varepsilon<c
$$

and this contradicts (6).

If $\varphi^{(1)}$ is not complete it follows from the lemma that we can find a positive integer $p$ such that, for each $q>p, q \in \mathbb{N}$, there exists $r \in \mathbb{N}, r>q$, and a subset $H$ of $\mathbb{N}$ such that

$$
\begin{gathered}
a_{n}^{(p)} \neq 0, \quad n \in H, \\
\inf \left\{\frac{a_{n}^{(p)}}{a_{n}^{q}}: n \in H\right\} \neq 0 \\
\inf \left\{\frac{a_{n}^{(p)}}{a_{n}^{(r)}}: n \in H\right\}=0 .
\end{gathered}
$$


We put $q=q_{1}, r=q_{2}$. We determine an increasing sequence of natural numbers

$$
n_{11}<n_{12}<\ldots<n_{1 i}<\ldots
$$

such that

$$
\lim _{i \rightarrow \infty} \frac{a_{n_{11}}^{(p)}}{a_{n_{1 i}}^{\left(q_{1}\right)}}=c_{1} \neq 0, \quad \lim _{i \rightarrow \infty} \frac{a_{n_{11}}^{(p)}}{a_{n_{1 i}}^{\left(a_{2}\right)}}=0 .
$$

Let us suppose that, for $s=1,2, \ldots, m$, sequences of positive integers $n_{s 1}<n_{s 2}<$ $\ldots<n_{s i}<\ldots$ and numbers $q_{s}, q_{s+1}$ in $\mathbb{N}$ have already been constructed such that

$$
\begin{aligned}
& \lim _{i \rightarrow \infty} \frac{a_{n_{i t}}^{(p)}}{a_{n_{i}}^{\left(q_{i}\right)}}=c_{s} \neq 0, \\
& \lim _{i \rightarrow \infty} \frac{a_{n_{i s}}^{(p)}}{a_{n_{s i}}^{\left(q_{s+1}\right)}}=0 .
\end{aligned}
$$

Starting from $q_{m+1}$ we can find a positive integer $q_{m+2}>q_{m+1}$ and a subset $P$ of $\mathbb{N}$ such that

$$
\begin{aligned}
& \inf \left\{\frac{a_{n}^{(p)}}{a_{n}^{\left(q_{m+1}\right)}}: n \in P\right\} \neq 0 \\
& \inf \left\{\frac{a_{n}^{(p)}}{a_{n}^{\left(a_{m+2}\right)}}: n \in P\right\}=0 .
\end{aligned}
$$

By (9), we can assume that $P$ has been chosen disjoint from

$$
\left\{n_{s i}: s=1,2, \ldots, m ; i=1,2, \ldots\right\} \text {. }
$$

Now we select a sequence

$$
n_{(m+1) 1}<n_{(m+1) 2}<\ldots<n_{(m+1) i}<\ldots
$$

in $P$ such that

$$
\begin{aligned}
& \lim _{i \rightarrow \infty} \frac{a_{n_{(m+1)}}^{(p)}}{a_{n_{(m+1)}}^{\left(a_{m+1}\right)}}=c_{m+1} \neq 0, \\
& \lim _{i \rightarrow \infty} \frac{a_{n_{(m+1)}}^{(p)}}{a_{n_{(m+1)}}^{\left(a_{m+2}\right)}}=0 .
\end{aligned}
$$

Let $\Lambda$ be the sectional subspace of $\lambda$ defined by the system of steps

$$
B^{(m)}=\left(b_{i j}^{(m)} ; i, j=1,2, \ldots\right) \quad m=1,2, \ldots
$$

where

$$
\begin{aligned}
b_{i j}^{(m)} & =a_{n_{j}+1}^{\left(a_{m+1}\right)}, \quad m=2,3, \ldots \\
b_{i j}^{(1)} & =a_{n_{j}}^{(p)} .
\end{aligned}
$$

Theorem 2. If $\varphi^{(1)}$ is not complete, $\lambda^{x}\left[\beta\left(\lambda^{x}, \lambda\right)\right]$ does not fulfil the Mackey convergence condition. 
Proof. Obviously, it is enough to show that $\Lambda^{x}\left[\beta\left(\lambda^{x}, \Lambda\right)\right]$ does not verify the Mackey convergence condition. In $\Lambda^{x}$ we consider the sequence $\left(\left(x_{i j}^{(n)}\right)\right)_{n=1}^{\infty}$ defined in the following way:

$$
\begin{aligned}
& x_{1 j}^{(n)}=0, \quad j=1,2, \ldots ; \quad n=1,2, \ldots \\
& x_{i j}^{(n)}=0, \quad j=1,2, \ldots n ; \quad i=2,3, \ldots \\
& x_{i j}^{(n)}=\frac{1}{i} b_{i j}^{(1)}, \quad j=n+1, n+2, \ldots ; \quad i=2,3, \ldots
\end{aligned}
$$

Let us see first that the sequence $\left(\left(x_{i j}^{(n)}\right)\right)_{n=1}^{\infty}$ converges to the origin in $\Lambda^{x}\left[\beta\left(\Lambda^{x}, \Lambda\right)\right]$. If $B$ is a bounded set in $\Lambda\left[\mu\left(\Lambda, \Lambda^{x}\right)\right]$ there exists a sequence $\left(h_{m}\right)$ of positive numbers such that, for each $\left(u_{i j}\right) \in B$.

$$
\sum_{i, j=1}^{\infty}\left|u_{i j}\right| b_{i j}^{(m)} \leqq h_{m}, \quad m=1,2, \ldots
$$

Given a positive number $\varepsilon$, we can find $i_{0} \in \mathbb{N}, i_{0} \geqq 2$, such that

$$
\frac{h_{1}}{i_{0}}<\frac{\varepsilon}{2}
$$

Then, for each $\left(u_{i j}\right) \in B$ and $n \in N$, it follows that

$$
\begin{aligned}
\left|\sum_{i, j=1}^{\infty} u_{i j} x_{i j}^{(n)}\right| & \leqq\left|\sum_{i=2}^{i_{0}} \sum_{j=1}^{\infty} u_{i j} x_{i j}^{(n)}\right|+\sum_{i=i_{0}+1}^{\infty} \sum_{j=1}^{\infty}\left|u_{i j}\right| \frac{1}{i} b_{i j}^{(1)} \\
& \leqq\left|\sum_{i=2}^{i_{0}} \sum_{j=1}^{\infty} u_{i j} x_{i j}^{(n)}\right|+\frac{1}{i_{0}} h_{1} \leqq\left|\sum_{i=2}^{i_{0}} \sum_{j=1}^{\infty} u_{i j} x_{i j}^{(n)}\right|+\frac{\varepsilon}{2}
\end{aligned}
$$

We can put (9) in the form

$$
\lim _{i \rightarrow \infty} \frac{b_{i j}^{(1)}}{b_{i j}^{(i)}}=0, \quad i=2,3, \ldots
$$

and, therefore, there exists $j_{0} \in \mathbb{N}$ such that

$$
\frac{b_{i j}^{(1)}}{b_{i j}^{(i)}}<\frac{\varepsilon}{2 i_{0} h_{i}}, \quad j \geqq j_{0}, \quad i=2,3, \ldots, i_{0}
$$

Then, if $n>j_{0}$ it follows for $\left(u_{i j}\right) \in B$,

$$
\begin{aligned}
\left|\sum_{i, j=1}^{\infty} u_{i j} x_{i j}^{(n)}\right| & \leqq\left|\sum_{i=2}^{i_{0}} \sum_{j=1}^{\infty} u_{i j} x_{i j}^{(n)}\right|+\frac{\varepsilon}{2} \\
& \leqq \sum_{i=2}^{i_{0}} \sum_{j=j_{0}+1}^{\infty}\left|u_{i j}\right|\left|x_{i j}^{(n)}\right|+\frac{\varepsilon}{2} \leqq \sum_{i=2}^{i_{0}} \sum_{j=j_{0}+1}^{\infty}\left|u_{i j}\right| \frac{b_{i j}^{(1)}}{i}+\frac{\varepsilon}{2} \\
& \leqq \sum_{i=2}^{i_{0}} \frac{\varepsilon}{2 i_{0} h_{i}} \sum_{j=i_{0}+1}^{\infty}\left|u_{i j}\right| b_{i j}^{(i)}+\frac{\varepsilon}{2} \leqq \sum_{i=2}^{i_{0}} \frac{\varepsilon}{2 i_{0} h_{i}} h_{i}+\frac{\varepsilon}{2} \leqq \varepsilon,
\end{aligned}
$$

hence $\left(\left(x_{i j}^{(n)}\right)\right)_{n=1}^{\infty}$ converges to the origin in $\Lambda^{x}\left[\beta\left(\Lambda^{x}, \Lambda\right)\right]$. 
On the other hand, given the natural number $m$, by (8) it follows that

$$
\lim _{j \rightarrow \infty} \frac{x_{(m+1) j}^{(n)}}{b_{(m+1) j}^{(m)}}=\frac{1}{m+1} \lim _{j \rightarrow \infty} \frac{b_{(m+1) j}^{(1)}}{b_{(m+1) j}^{(m)}}=\frac{1}{m+1} c_{m+1} \neq 0, \quad n=1,2, \ldots
$$

so that $\left(\left(x_{i j}^{(n)}\right)\right)_{n=1}^{\infty}$ does not converge to the origin in $\Lambda_{\beta^{(m)}}^{x}$.

From the former results we obtain the following theorem:

Theorem 3. In an echelon $\lambda$, the following conditions are equivalent:

1. $\varphi^{(1)}$ is complete.

2. $\lambda\left[\mu\left(\lambda, \lambda^{x}\right)\right]$ is quasi-normable.

3. $\lambda^{x}\left[\beta\left(\lambda^{x}, \lambda\right)\right]$ verifies the Mackey convergence condition.

In (1) A. Grothendieck asks if there exists a space with a countable fundamental system of bounded sets which verifies the Mackey convergence condition but not the strict Mackey convergence condition. In (5) S. Warner gives an example answering this question. In the following proposition we give a class of spaces with this property.

Proposition. If $E$ is a Banach space such that $E^{\prime}\left[\sigma\left(E^{\prime}, E\right)\right]$ is not separable there is a topology $\mathbb{E}$ on $E$, compatible with the dual pair $\left\langle E, E^{\prime}\right\rangle$, such that $E[\&]$ is a (DF)-space which verifies the Mackey convergence condition but not the strict Mackey convergence condition.

Proof. Let $\mathbb{E}$ be the topology on $E$ of the uniform convergence on every countable

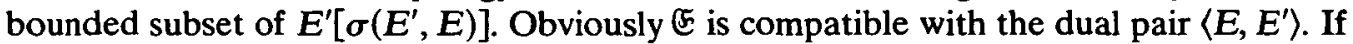
$B$ is the closed unit ball of $E$, a fundamental system of bounded set of $E[\mathcal{E}]$ is given by

$$
\{n B: n=1,2, \ldots\}
$$

Every locally convex space $E_{n B}$ coincides with $E_{B}, n=1,2, \ldots$ and, since $E^{\prime}\left[\sigma\left(E^{\prime}, E\right)\right]$ is not separable, $E$ is different of the topology of $E$, hence $E$ does not coincide with the topology of $E_{n B}$ on $B$ and, therefore, $E[\&]$ does not verify the strict Mackey convergence condition.

Since $E[\mathbb{E}]$ is, obviously, a $(D F)$-space, $F$ and $\beta\left(E, E^{\prime}\right)$ (which coincides with the topology of $E$ ), coincide on every countable subset of $E[\mathcal{E}],[3$, p. 392] and therefore if $\left(x_{n}\right)$ is a sequence in $E[\&]$ which converges to $x$, it also converges to $x$ in $E=E_{B}$.

Question. Is it true that every separable Fréchet space, such that its strong dual verifies the Mackey convergence condition, is quasi-normable?

Theorem 4. If $\lambda\left[\mu\left(\lambda, \lambda^{x}\right)\right]$ is not quasi-normable, it is the strong dual of a (LB)space which is not complete.

Proof. The space $\lambda\left[\mu\left(\lambda, \lambda^{x}\right)\right]$ is the strong dual of the subspace $\varphi$ of $\lambda^{x}\left[\beta\left(\lambda^{x}, \lambda\right)\right]$ (2), hence $\lambda\left[\mu\left(\lambda, \lambda^{x}\right)\right]$ is the strong dual of $\varphi^{(1)}$. On the other hand, $\varphi^{(1)}$ is a (LB)-space, (4), and, by Theorem $1, \varphi^{(1)}$ is not complete. 
Corollary. If $\lambda\left[\mu\left(\lambda, \lambda^{x}\right)\right]$ is not distinguished, it is the strong dual of a non-complete (LB)-space.

Proof. It is straightforward considering Theorem 4 and the fact that every quasinormable Fréchet space is distinguished. (3, p. 399).

Acknowledgement. I would like to thank the referee for his comments.

\section{REFERENCES}

(1) A. Grothendieck, Sur les espaces (F) et (DF), Summa Brasil, Math. 3 (1954) 57-123.

(2) T. Komura and Y. Komura, Sur les espaces parfaits de suites et leurs généralisations, $J$. Math. Soc. Japan 15 (1963) 319-338.

(3) G. KötHE, Topological Vector Spaces I (Springer-Verlag, Berlin-Heilelberg-New York, 1969).

(4) M. Valdivia, Algunas propiedades de los espacios escalonados, Rev. Real Acad. Ciencias, Madrid, 73 (1979) 389-400.

(5) S. WARNER, The topology of compact convergence on continuous functions spaces, Duke Math. J. 25 (1958) 265-282.

Facultad de Matemáticas

Dr MOLINER, $\mathbf{s} / \mathbf{n}$

Burjasot, Valencia

SPAIN 